piv

1972

cos

1949

CHMREF
SMALL WONDERS 



\section{SMAH WONDERS}

Puppets and Marionettes

THE COOPER UNION MUSEUM

FOR THE ARTS OF DECORATION COOPER SQUARE, NEW YORK 


\section{CATALOGUE}

STRING MARIONETTES

1. Appetite $(\operatorname{dog})$; made by Gayle and Doug Anderson, United States, 20 th century.

2. Gertrude; Gayle and Doug Anderson, United States, 20th century.

Lent by Gayle and Doug Anderson

3. Gossip, Spinning wheel; Bil Baird, United States, 20th century.

4. Gramps; Bil Baird, United States, 20th century.

5. Egyptian; Bil Baird, United States, 20th century.

6. CalfF; Bil Baird, United States, 20th century.

Lent by Bil Baird's Marionettes

7. Wizard; Stephen Lowe, United States, 20th century.

Lent by Berkeley Marionettes, Inc.

8. Fairy Godmother; Remo Bufano, United States, 20th century.

9. The Don; Remo Bufano, United States, 20th century.

Lent by Remo Bufano Studios, Inc.

10. Group of Commedia dell' Arte FigurEs; Italy, 18th century. The Cooper Union Museum

11. EuIsey; Basil Milovsoroff, United States, 20th century.

12. Kumashka Yashka; Basil Milovsoroff, United States, 20th century.

Lent by the Folktale Puppet Studio

13. Harlequin; Carroll French, United States, 20th century.

14. Pierrot; Carroll French, United States, 20th century.

15. Columbine; fintrghofifench, United States, BdIl century. AN

1. FEB 181998

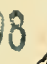

LIBRARIES
16. Box OfIs; Carroll French, United States, 20th century.

17. The Superior Puppet; Carroll French, United States, 20th century.

Lent by Carroll French

18. Dancer; Sue Hastings, Inc., United States, 20th century.

19. Four characters from Rip Van Winkle; Sue Hastings, Inc., United States, 20th century. Lent by Sue Hastings, Inc.

20. Fisherman; Spain, 19th century.

21. Самво, Тову (?); United States, late 19 th century.

22. Lady Margaret; Tony Sarg Studio, United States, 20th century.

23. Dynamic Design; Bil Baird, United States, 20th century.

24. TAR BABY; Tony Sarg Studio, United States, 20th century.

25. George Washington; Cedric and Mabel Head, United States, 20th century.

26. Tony Sarg Portrait Puppet; Lillian Owen, United States, 20th century.

27. LADY; India, 19th century.

Lent by the Kingsland Collection, courtesy of Briggs Management

28. Wiтch Hazel; Caroline and Otto Kunze, United States, 20th century.

29. Book Worm; Caroline and Otto Kunze, United States, 20th century.

30. Lester the Jester; Caroline and Otto Kunze, United States, 20th century.

Lent by Otto Kunze Marionette Productions

31. Caliph; Jero Magon, costume by Esphyr Slobodkina, United States, 20th century. Lent by Jero Magon 
32. Woman; France, 18th century.

33. Children's Puppets; Mexico, 20th century.

34. Ghost; North China, 20th century.

35. LADY; Japan, 20th century.

Lent by Brander Mathews Dramatic Museum

36. Bенат ; George Nellé, United States, 20th century.

Lent by George Nellé of the Marionette Repertory Theater

37. Joe Crocus; Mildred May Osgood, United States, 20th century.

38. Royal Pet; Mildred May Osgood, United States, 20th century.

39. Flower FaIRy; Mildred May Osgood, United States, 20th century.

40. Tree; Mildred May Osgood, United States, 20th century.

41. Flower; Mildred May Osgood, United States, 20th century.

42. Sir Green-up; Mildred May Osgood, United States, 20th century. Lent by Mildred May Osgood

43. Katherine Dunham; Frank Paris, United States, 20th century. Lent by Frank Paris

44. Caliban; Margo and Rufus Rose, United States, 20th century.

45. Oriental Dancers; Margo and Rufus Rose, United States, 20th century.

Lent by Rufus Rose Marionettes

\section{HAND PUPPETS}

46. Punch; made by Bil Baird, United States, 20th century.

Lent by Bil Baird's Marionettes

47. MaN, Woman, Two "PUNCH" TYPES; Remo Bufano, United States, 20th century.

Lent by Remo Bufano Studios, Inc.

48. Scaramouche; Italy (?), 19th century.
49. MaN; Italy (?), 19th century. The Cooper Union Museum

50. Kung-SeE; Alice E. Fox, United States, 20th century.

51. The Mandarin; Alice E. Fox, United States, 20th century.

Lent by Mrs. Alice E. Fox

52. Jolly Punch, Devil, Hangman AND Doctor; Spain, 20th century.

53. Punch; England, 18th century (?).

54. Flunky, Doctor, Brigella, ColUMBINE; Italy, 18th century.

Lent by Kingsland Marionette Collection, courtesy of Briggs Management

55. Man, Woman; Anatole, France, 19th century.

56. Policeman; Anatole, France, 19th century.

57. Punch, Judy, Baby; England, 19th century.

Lent by Brander Mathews Dramatic Museum

58. HARRIET; Kurt Seligmann, United States, 20th century.

Lent by Tibor Nagy Marionettes

\section{ROD PUPPETS}

59. MAN; China, 20th century.

60. MAN (?); Java.

Lent by The American Museum of Natural History

61. The Applauder; made by Bil Baird, United States, 20th century. Lent by Bil Baird's Marionettes

62. MaN; Italy, 18th century. The Cooper Union Museum

63. Ballet DANCER (from chorus?); Italy, 19th century. Lent by Carroll French

64. Rinaldo; Sicily, 19th century. Lent by Katherine Gravet

65. Orlando; Giovanni Grasso (?), Sicily, 19th century. 
66. Woman; Armenia, late 19th century.

67. Clown; China, 20th century.

68. WAyang GoleK type; Java, 19th century.

Lent by Kingsland Marionette Collection, courtesy of Briggs Man. agement

69. Carlo Martello; Agrippino (Papa) Manteo, United States, 20th century.

Lent by The Manteo Family

70. Violetta; Esther T. Wolff, United States, 20th century.

71. Escamillo; Esther T. Wolff, United States, 20th century.

Lent by Esther T. Wolff

\section{SHADOW PUPPETS}

72. KoembaKarma; Bali, 20th century.

73. I SANgoet; Bali, 20th century.

74. TJondong; Bali, 20th century.

75. RangdA (witch); Bali, 20th century.

Lent by The American Museum of Natural History

76. WARRIOR AND LADY; made by Chinese craftsmen, Peiping, China.

77. Man in Procession, Lady; Chinese craftsmen, Shansi Province, China.

78. Prime Minister; Chinese craftsmen, Szechuan Province, China.

79. Peach Tree; Chinese craftsmen, China.

80. LADY; Chinese craftsmen, Luanchow, China, 20th century.

81. Calf; Pauline Benton, United States, 20th century.

82. Karogöz, The Armenian; Turkish craftsmen, Turkey, 20th century.

83. WomAn; Siamese craftsmen, Siam.

Lent by Pauline Benton and The Red Gate Players
84. The Wolf, Mulan, Jolly Courtier, Chinese Gentlemen; Chinese craftsmen, China, 19th century.

85. Chinese Emperor in FUll ARMor oN HORSEBACK; Chinese craftsmen, China, late 19th century.

86. Chinese General IN FULL ARMoR; Chinese craftsmen, China, late 19th century.

87. The White Elephant pulling the Peking BRIdal cart; Chinese craftsmen, China, late 19th century.

Lent by Dr. Theodore Bodde

88. GENERAL; Chinese craftsmen, West China, 19th century.

89. A Lady General; Chinese craftsmen, West China.

90. Butterfly; Chinese craftsmen, West China, 19th century.

91. Rat; Chinese craftsmen, West China, 19th century.

92. BEE; Chinese craftsmen, West China, 19th century.

93. Crane; Chinese craftsmen, West China, 19th century.

94. OWL; Chinese craftsmen, West China, 19th century.

95. 3 WAJANG FIGURES, FRAMEd; Javanese craftsmen, Java, 19th century.

96. The Prince greets Cinderella; probably Théâtre Séraphin, France, 19th century.

97. Cinderella during the Ball; probably Théâtre Séraphin, France, 19th century.

98. Don Quixote and the Peasant; probably Théâtre Séraphin, France, 19th century.

99. Don Quixote atTacking an IMAGINARY FOE; probably Théâtre Séraphin, France, 19th century. 
100. Group of Figures From Madame la Baronne; probably Théâtre Séraphin, France, 19th century.

101. Tree, AND Box; probably Théâtre Séraphin, France, 19th century.

The Cooper Union Museum

102. Monkey-God Panel; Siam, 18th century (?).

103. GARODA BiRD; Java, 19th century. Lent by Kingsland Collection, courtesy of Briggs Management

104. 3 Musicians; Jero Magon, United States, 20th century.

105. Marco Polo; Jero Magon, United States, 20th century.

106. Ghost of Hamlet's Father; Jero Magon, United States, 20th century.

Lent by Jero Magon

107. Woman; France, 19th century. Lent by Brander Mathews Dramatic Museum

\section{FINGER AND GLOVE PUPPETS}

108. Dancer; Tony Sarg, United States, 20th century.

Lent by Kingsland Marionette Collection, courtesy of Briggs Management

109. Russian Dancer; Jero Magon, United States, 20th century. Lent by Jero Magon

110. Bull, the Frog; Hallie C. Patingale, United States, 20th century. Lent by Hallie C. Patingale

PRINTS AND DRAWINGS

111. OEdipus Rex; Robert Edmond Jones, United States, 20th century.

Lent by Remo Bufano Studios, Inc.

112. Punch and Judy on Boston Common; Lagarde, United States, 19th century.

The Cooper Union Museum
113. The Puppeteer; Alfredo Zalce, Mexico, 20th century. Lent by Alleine E. Dodge

114. Rip Van Winkle, Alice in Wonderland, Tony Sarg, United States, 20th century.

Lent by Kingsland Marionettes Collection, courtesy of Briggs Management

115. Man with Rod Puppet; Japan, 19th century.

Lent by Brander Mathews Dramatic Museum

116. The Story of Punch: Characters from Punch and Judy Plays; W. P. A. Artists, United States, 20th century.

Lent by Index of American Design, National Gallery of Art

117. BEast, costume design; George Nellé, United States, 20th century.

118. Beauty and the Beast Set; Robert Pryor, United States, 20th century.

Lent by George Nelle of the Marionette Repertory Theater

119. Cook, Costume sketch; Bernard Pfriem, United States, 20th century.

Lent by Tibor Nagy Marionettes

\section{ADDITIONAL MATERIAL}

120. Wolf, Red Riding Hood, GrandMOTHER, WoOdsman, Stop Motion Puppets; Remo Bufano, United States, 20th century.

Lent by Remo Bufano Studios, Inc.

121. Ombres Chinoises; Book of Scripts, probably Théâtre Séraphin, France, 19th century. The Cooper Union Museum

122. Le Théâtre Italien ou Le RecuEIL de Toutes les Scénes; Book, Geneva, 17th century. Lent by Katherine Gravet

123. Mr. Punch, Sign; England, 19th century. 
124. Man; Hand Control Puppet, Japan, 18th century.

125. Puppet Panel; Java, 20th century.

126. Puppet Panel; Bali, 19th century.

Lent by Kingsland Marionette Collection, courtesy of Briggs Management
127. Man; Home Puppet, China, 20th century.

Lent by Brander Mathews Dramatic Museum

128. Prince, undressed; George Nellé, United States, 20th century.

129. Puppet castings; George Nellé, United States, 20th century. Lent by George Nelle of the Marionettes Repertory Theater

\section{REFERENCES}

\section{In the Libraries of the Cooper Union Relating to Puppets, Marionettes and Shadow-Plays}

Ombres Chinoises. Paris 1810-12. 2 vols. Manuscript librettos probably for the Théâtre Séraphin.

Ombres Chinoises. Musical parts, arranged for two violins, alto and basso. Paris, 1800? 4 vols. Engraved, with manuscript additions. Probably for use in the Théâtre Séraphin.

Aghion, MAx. Le théâtre à Paris au XVIIIle siècle. Paris, Librairie de France, n.d. 442 p. Marionnettes, p. 238.

Allemagne, Henry René d'. Histoire des jouets. Paris, Hachette, 1902. 316 p. Les marionnettes, p. 238-63; Les ombres chinoises, p. 264-72.

Altherr, Alfred. Marionetten. ErlenbachZürich, Rentsch, 1926? 49 p.

Auroy, Mlle. B. Ainsi font les marionnettes! Paris, Nathan, 1930.

Bapst, Germain. Essai sur l'histoire du théâtre. Paris, Hachette, 1893. 693 p. Marionnettes, p. 17, 53-5, 275, 345, 377, 480.

Batchelder, Marjorie Hope. The puppet theatre handbook. New York, Harper, 1947. 293 p.

Beaumont, Cyril William. Puppets and the puppet stage. New York, Studio, 1938. $144 \mathrm{p}$.

Boenn, Max von. Dolls and puppets. London, Harrap, 1932. 521 p.

Bufano, Remo. Be a puppet showman. New York, Century, 1933. 168 p.

Bussell, JAN. Marionettes: how to make them. Ditchling, Sussex, Pepler \& Sewell, 1934. $28 \mathrm{p}$.
Bussell, John Garrett. The puppet theatre. London, Faber \& Faber, 1946. 143 p.

Chronicle of the Museum for the Arts of Decoration of the Cooper Union. v. 1 , no. 1, Winter 1934-35. Material relating to the small theatre, p. 23-30.

LE Cinéma, des origines à nos jours. Paris, Édilions du cygne, 1932. 366 p. Ombres chinoises, p. 25-34.

Croztère, Alphonse. Le vrai théâtre des enfants. Paris, Nathan, 1934. 127 p.

Darjou, A. Les silhouettes faciles, ombres amusantes. Paris, Journal amusant, n.d. $20 \mathrm{pl}$.

Duranty, Louis Émile Édouard. Théâtre des marionnettes $\mathrm{du}$ jardin des Tuileries. Paris, Dubuisson, 1864. 387 p.

Enciclopedia Italiana. Rome, Istituto Giovanni Treccani, 1929-39. v. 22. Marionetta, p. 356-8.

Encyclopedia Britannica. 14th ed. New York, 1932. v. 14. Marionettes, p. 906-7.

Eudel, Paul. Les ombres chinoises de mon père. Paris, Rouveyre, 1885. $306 \mathrm{p}$.

FEU SÉraphin, histoire de ce spectacle depuis son origine jusqu'à sa disparition, 17761870. Lyon, Scheuring, 1875. $334 \mathrm{p}$.

Ficklen, Bessie. A handbook of fist puppets. New York, Stokes, 1935. 329 p.

Field, Marshall \& Co., Chicago. Exhibition of puppets and marionettes. Chicago, 1933. 23 p.

Field Museum of Natural History, Chicago. Dept. of Anthropology. Guide, part I. Oriental theatricals, by Berthold Laufer. Chicago, 1923. The shadow-play, China, p. $36-42$. 
Fleury, Jules. Souvenirs des funambules. Paris, Lévy, 1859. 320 p.

Fovquier, Marcel. Paris au XVIIIe siècle: ses divertissements-ses moeurs-directoire et consulat. Paris, Emile-Paul, n.d. 146 p. Marionnettes, p. 46-7.

Fournel, François Victor. Le vieux Paris: fêtes, jeux et spectacles. Tours, Mame, 1887. Marionettes . . . ombres chinoises, p. 290-334.

Freeman, Ruth. Cavalcade of toys. Watkins Glen, N. Y., Century House, 1942. 399 p. Shadowgraphy, p. 231-2.

Geist, Hans Friedrich. Spielzeug. Leipzig, Staackmann, 1938. 42 pl. Kasperle und Marionette, pl. 42.

Ginisty, Paul. France d'antan; le théâtre de la rue. Paris, Morancé, 1925. 61 p. Marionnettes, p. 29-30; Les Guignol, p. 48-50.

Green, Dana Saintsbury. Puppet making. London, Studio, 1935. 64 p.

GRöBER, KARL. Kinderspielzeug. Berlin, Deutscher Kunstverlag, 1928. 67 p. Marionetten, pl. 277-80, XI.

Hinot, Claude. Le fils à Guignol. Paris, Larousse, 188-?

Jackson, Emily. Toys of other days. London, Country Life, 1908. 308 p. Marionettes, Punch and Judy, p. 167-93; Shadowgraphy, p. 231-4.

JeAnne, PAul. Les théâtres d'ombres à Montmartre de 1887 à 1927. Paris, Editions des presses modernes, 1937. $173 \mathrm{p}$.

Joseph, Mrs. Helen. A book of marionettes. New York, Viking, 1929. 248 p.

Kennard, Joseph Spencer. Masks and marionettes. New York, Macmillan, 1935. 129 p.

Lemercier de Neuville, Louis. Les pupazzi noirs. Paris, Mendel, 1896? 304 p.

Lemercier de Neuville, Louis. Souvenirs d'un montreur de marionnettes. Paris, Bauche, 1911. 348 p.

MacIsaAc, Frederick John. The Tony Sarg marionette book. New York, Viking, 1932. $57 \mathrm{p}$.

McPharlin, Paul. Guide to puppet plays. Birmingham, Mich., 1932. 16 p. (Puppetry handbooks III).

McPharlin, Paul. The puppet theatre in America. New York, Harper, 1949. 506 p.

McPharlin, Paul. A repertory of marionette plays. New York, Viking, 1929. 372 p.

Magnin, Charles. Histoire des marionnettes en Europe. Paris, Lévy, 1852. 346 p.
MaLík, Jan. Puppetry in Czechoslovakia. Prague, Orbis, 1948. $56 \mathrm{p}$.

Mills, Mrs. Winifred. Marionettes, masks and shadows. Garden City, N. Y., Doubleday, 1928. $270 \mathrm{p}$.

Miyajima, Tsunao. Contribution à l'étude du théâtre japonais de poupées. Osaka, 1931. $109 \mathrm{p}$.

Munger, Martha Perrine. The book of puppets. Boston, Lothrop, 1934. 206 p.

The New International Encyclopedia. Second ed. New York, Dodd, Mead, 1916. Puppet, v. 19, p. 378-9; Shadow play, v. 20 , p. 768.

New York (City) Board of Education. Puppetry in the curriculum. New York, 1947. $171 \mathrm{p}$.

Peter Pry's puppet show. Philadelphia, Morgan \& son, n.d. 2 vols.

Pougin, Arthur. Dictionnaire historique et pittoresque du théâtre. Paris, FirminDidot, 1885. 775 p. Marionnettes, p. 449502; Ombres chinoises, p. 550.

Puppetry, a yearbook of puppets \& marionettes. Detroit, Mich., 1934, 1935, 1937, 1946-7. 4 vols. (Edited by Paul McPharlin).

Puppetry Bulletin. Birmingham, Mich., 1938-42. 4 vols. (Edited by Paul McPharlin).

Sadoul, Georges. Histoire générale du cinéma. I. L'invention du cinéma, 18321897. Paris, Editions Denoël, 1945. 362 p. Ombres chinoises, p. 199-201.

Seyffert, Oskar. Toys. Berlin, Wasmuth, 1922? 40 pl. Puppets, pl. 40 .

SÉzan, Claude. Les poupées anciennes. Paris, Éditions pittoresques, 1930. 196 p. La vie des marionnettes, p. 126-83.

LES SPECTACLES à travers les âges; théâtre, cirque, music-hall. Paris, Éditions du cygne, 1931. 366 p. Les marionnettes, p. 264; Théâtre d'ombres, p. 361.

Starr, Laura B. The doll book. New York, Outing pub. co., 1908. 238 p. Puppets and marionettes, p. 31-44.

Theatre Arts Monthly, v. 12, no. 7, July 1928. The marionette.

U. S. Works Progress Administration. Recreation Project, New York City. Puppet teaching news bulletin, v. 2, no. 6, July 1938-v. 3, no. 2, Summer 1939. 7 nos.

U. S. Works Progress Administration. Recreation Project, New York City. Puppetry manual. New York, 1938? 421.

Wimsatt, Genevieve Blanche. Chinese shadow shows. Cambridge, Mass., Harvard, 1936. $68 \mathrm{p}$.

Richard E. Morse. 


\section{ACKNOWLEDGMENT}

The Museum has received many helpful suggestions and much valuable information from the following:

Miss Pauline Benton

Carroll French

Mabel and Cedric Head

Leo Manteo

Mrs. Marjorie Batchelder McPharlin

George Nellé

Mrs. Frances Schram

Miss Bella Weitzner

Dr. Henry W. Wells

Grateful thanks for their assistance are given to these friends, and to the following lenders of puppets and related material:

American Museum of Natural History

Gayle and Doug Anderson

BIL BAIRD

Pauline Benton and the Red Gate Players

Berieley Marionettes, Inc.

Dr. Theodore Bodde

Remo Bufano Studios, Inc.

Miss Alleine E. Dodge

Alice E. Fox

Carroll French

Miss Katherine Gravet

Sue Hastings, Inc.

Kingsland Marionette Collection, courtesy of Briggs Management

Otto Kunze Marionette Productions

The Manteo Family

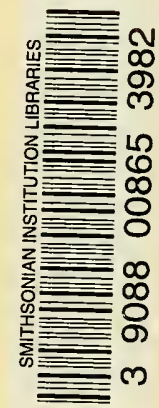

Brander Mathews Dramatic Museum,

Columbia University

Basil Milovgoroff and the Folktale Puppet Studio

Index of American Design, National Gallery of Art

George Nellé and the Marionette Repertory Theater

Mildred May Osgood

Frank Paris

Mrs. Hallie C. Patingale

Rufus Rose Marionettes

Tibor Nagy Marionettes

Esther Wolff and the Puppet Opera Company 

